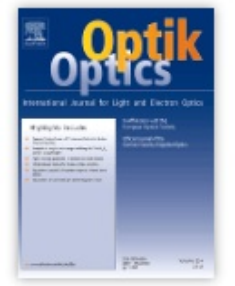

ISSN: $0030-4026$

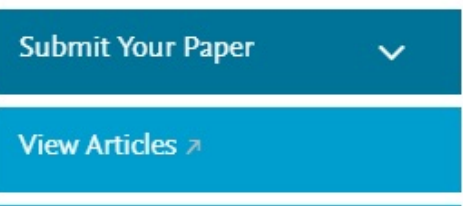

Guide for Authors

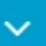

Abstracting/ Indexing

Track Your Paper

$\checkmark$

Order Journal

\section{Sample Issue $\pi$}

Journal Metrics 즈

CiteScore: 1.03 (1)

More about CiteScore $\lambda$

Impact Factor: 0.835 (1)

5-Year Impact Factor: 0.781 (1)

Source Normalized Impact per Paper (SNIP): 0.703 (i)

SCImago Journal Rank(SJR): 0.307 (1)

$>$ View More on Journal Insights $\lambda$

Related Links 주

$>$ Author Stats $\pi$ (i)

$>$ Publishing Campus $\lambda$

$>$ Author Services

$>$ Try out personalized alert

features

\section{Looking to uncover more} research tailored to you?

Register now to create a free account (we only ask for a few personal details).

ScienceDirect

\section{Optik}

International Journal for Light and Electron Optics

\section{$>$ Supports Open Access}

Editor-in-Chief: Prof. Dr. Theo Tschudi

$>$ View Editorial Board

\section{> Elsevier Physics homepage}

Optik publishes articles on all subjects related to light and electron optics and offers a survey on the state of research and technical development within the following fields:

Optics:

- Optics design, geometrical and beam optics, wave optics

- Optical and micro-optical components, diffractive...

Read more

\section{Most Downloaded Recent Articles Most Cited Open Access Articles}

Photocatalytic reaction and degradation of methylene blue on $\mathrm{TiO}_{2}$ nano-sized particles $\pi$ R.S. Dariani |A. Esmaeili|...

Investigation on factors to influence color emotion and color preference responses $\lambda$ Rui Gong $\mid$ Qing Wang $\mid$...

Higher oxidation level in graphene oxide $\pi$ Vaishali Gupta $\mid$ Neeraj Sharma $\mid .$.

View All Articles >

\section{Announcements}

Heliyon Partner Journal त

This journal is now partnering with Heliyon, an open access journal from Elsevier publishing quality peer reviewed research across all disciplines. Partner journals provide authors with an easy route to transfer their research to Heliyon.

$>$ Learn more at Heliyon.com $x$

Publish your data, software, materials or methods in brief, citable articles Some elements of the research cycle deserve more attention than they usually get. Think data, software, materials and methods - what happens to these when your research project is finished?

\section{$>$ View All}

\section{Special Issues}

Special issues published in Optik.

Optical Wireless Communication Systems $\pi$

Zabih Ghassemlooy | Stanislav Zvanovec |...

$>$ View All

\section{Call for papers}

Special Issue on $5^{\text {th }}$ Colloquium on Optical Wireless Communications (OWC)

$>$ View All 
Home > Journals > Optik > Editorial Board

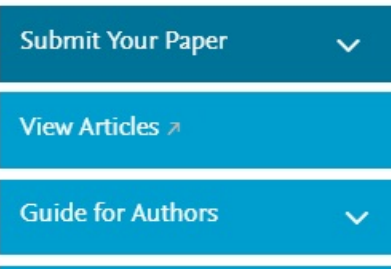

Abstracting/ Indexing

Track Your Paper

\section{$\checkmark$}

Order Journal

\section{Sample Issue $\pi$}

Journal Metrics

CiteScore: 1.03 (1)

More about CiteScore

Impact Factor: 0.835 (1)

5-Year Impact Factor: 0.781 (1)

Source Normalized Impact per Paper (SNIP): 0.703 (1)

SCImago Journal Rank(SJR): 0.307 (1)

$>$ View More on Journal Insights $\lambda$

\section{Related Links $\pi$}

$>$ Author Stats $\pi$ (1)

$>$ Publishing Campus $\pi$

$>$ Author Services

$>$ Try out personalized alert features

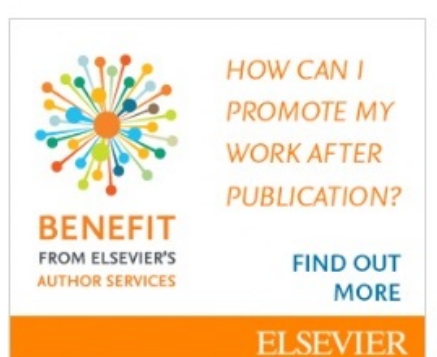

\section{Optik Editorial Board}

\section{Editor-in-Chief}

Prof. Dr. Theo Tschudi

Hasenberg 5, INOPTEC GmbH, 3210, Kerzers, Switzerland Email Prof. Dr. Theo Tschudi

\section{Section Editors}

\section{Fibre Optics}

H. Bartelt

Dept. of Fiber Optics, Institute of Photonic Technology, Jena, Germany Email H. Bartelt

Nonlinear Dynamics in Optics and Photonics, Optical Solitons

\section{A. Biswas}

Department of Physics, Chemistry and Mathematics, Alabama A \& M University, Alabama, USA

Email A. Biswas

Soliton perturbation; quasi-monochromatic solitons;

quasi-particle theory; non-Kerr laws; conservation laws; differential group delay; DWDM systems.

\section{Metamaterials Optic}

\section{P.K. Choudhury}

Inst. of Microengineering and Nanoelectronics, Universiti Kebangsaan Malaysia, Selangor, Malaysia

Email P.K. Choudhury

Thin Films and Plasmonics

\section{Faryad}

Department of Physics, Lahore University of Management Sciences (LUMS), Lahore, Pakistan Email M. Faryad

Electromagnetic surface waves, Dyadic Green functions, Photonic Crystals, Optical Metamaterials

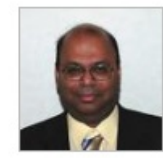




\section{Optical Networks}

\section{J. Healy}

School of Electrical and Electronic Engineering, University College Dublin, Dublin, Ireland

Email J. Healy

Numerics of optical propagation, holography, microscopy,

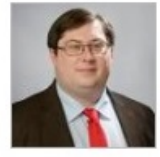

sampling theory, mathematical tools like linear integral transforms and phase space

Optical Design, Optical Metrology and

Interferometry, Speckle Metrology and Image

Processing

D.P. Kelly

Fakultät für Maschinenbau, Technische Universität Ilmenau, Ilmenau,

Germany

Email D.P. Kelly

\section{D Display}

J. Liu

School of Optoelectronics, Beijing Institute of Technology, 100081, China Email J. Liu

\section{Optical Networks}

\section{Zhao}

National Engineering Research Center for E-Learning (NERCEL), Central China Normal University, Wuhan, China Email L. Zhao

\section{Board}

\section{Prof. Dr. Hartmut Bartelt}

Dept. of Fiber Optics, Institute of Photonic Technology, Jena, Germany

Dr. Milivoj Belic

Quatar Campus, Texas A \& M University, Qatar

Prof. Dr. Eusebio Bernabeu

Department of Optics, Universidad Complutense de Madrid, Madrid, Spain

Prof. Dr. Shanti Bhattacharya

Dept. of Electrical Engineering, Indian Institute of Technology Madras, Chennai, India

\section{Professor Michael J. Damzen}

The Blackett Laboratory, Imperial College London, London, UK

\section{Prof. Dr. Francois Flory}

Institut Fresnel Dom. Univ. de St. Jerome, Ecole Nationale Superieure de Physique de Marseille, Marseille, France

Prof. Dr. Guy Indebetouw

Dept. of Physics, Virginia Polytechnic Institute \& State University, Blacksburg, Virginia, USA 
Prof. Dr. Henryk Kasprzak

Inst. of Physics, Wroclaw University of Technology, Wroclaw, Poland

Prof. Dr. Baida Lü

Inst. of Laser Physics and Laser Chemistry, Sichuan University, Chengdu, China

\section{Professor Akhlesh Lakhtakia}

Dept. of Engineering Science \& Mechanics, Pennsylvania State University, Pennsylvania, USA

\section{Prof. Dr. Kaoru Minoshima}

Graduate School of Informatics and Engineering, The University of ElectroCommunications, Tokyo, Japan

\section{Prof. Dr. Serguey Odoulov}

Institue of Physiks, National Academy of Sciences of Ukraine, Kiev, Ukraine

\section{Professor Yukitoshi Otani}

Center for Optical Research and Education, Utsunomiya University, Tochigi, Japan

Professor John Sheridan

Electronic \& Mechanical Engineering, University College Dublin, Dublin 4, UK

Dr. Serguej Stepanov

Dept. of Optica, CICESE, Ensenada, Mexico

Prof. Dr. Jari Turunen

Dept. of Physics, University of Eastern Finland, Joensuu, Finland

\section{Dr. Lingli Wang}

Leitung Forschung und Entwicklung, Jos. Schneider Optische Werke $\mathrm{GmbH}$, Bad Kreuznach, Germany

Prof. Dr. Chunming Zhang

School of Sciences, Xi'an Jiaotong University, China

Dr. Qin Zhou

School of Electronics and Information Engineering, Wuhan Donghu University, Wuhan, China 


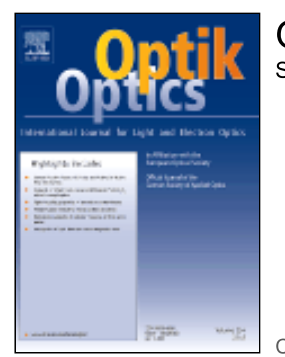

Optik - International Journal for Light and Electron Optics

Supports Open Access | About this Journal | Sample Issue Online | European Optical Society

Get new article feed

Get new Open Access article feed

Subscribe to new volume alerts

Add to Favorites

Copyright @ 2017 Elsevier GmbH. All rights reserved

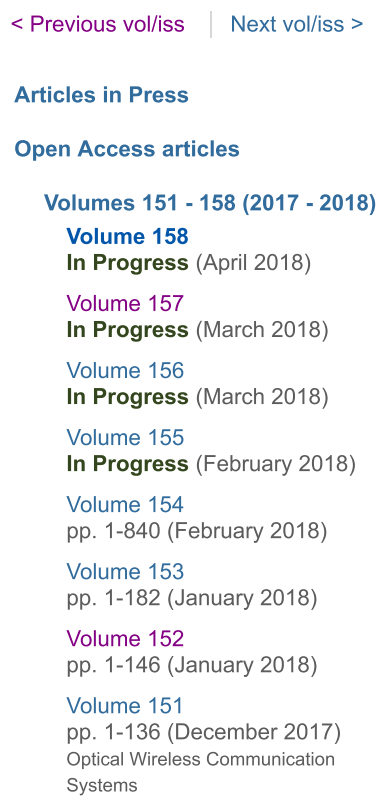

Articles in Press

Open Access articles

Volumes 151 - 158 (2017 - 2018)

Volume 158

In Progress (April 2018)

Volume 157

In Progress (March 2018)

Volume 156

In Progress (March 2018)

Volume 155

In Progress (February 2018)

Volume 154

pp. 1-840 (February 2018)

Volume 153

pp. 1-182 (January 2018)

Volume 152

pp. 1-146 (January 2018)

Volume 151

pp. 1-136 (December 2017)

Optical Wireless Communication

Systems

Volumes 141 - $150(2017)$

Volumes 131 - 140 (2017)

Volumes 121 - $130(2010$ - 2017)

Volumes 112 - 120 (2001 - 2009)
Optik - International Journal for Light and Electron Optics

Volume 158, In Progress (April 2018)

Articles 1 - 16

\begin{tabular}{|l|l|}
\hline \multicolumn{1}{|c|}{ Download PDFs } & Export \\
\hline $\begin{array}{l}\text { This issue is In Progress but contains articles that are final and fully citable. For } \\
\text { recently accepted articles, see Articles in Press. }\end{array}$
\end{tabular}

Original research articles

Perturbed optical solitons with spatio-temporal dispersion in $(2+1)$ dimensions by extended Kudryashov method Original Research Article Pages 1-14

Emrullah Yaşar, Yakup Yıldırım, Abdullahi Rashid Adem

Abstract $\quad$ PDF (1882 K)

Fabrication and characterization of helical long-period fiber gratings in single-mode fibers Original Research Article

Pages 28-32

Xingpeng Wang, Dongdong Wang, Qi Wang, Lunlun Xian, Li Li

Abstract $\quad$ PDF (1026 K)

Propagation characteristics of some intricate planar waveguides of practical interest by finite difference method Original Research Article Pages 15-27

Soumita Chakraborty, Prasenjit Ghosh, Somenath Sarkar Abstract $\quad$ PDF $(2030 \mathrm{~K})$

Reflective solar blind filter based on dielectric multilayer Original Research Article

Pages 33-36

Xiaodong Wang, Bo Chen, Ling Yao

Abstract $\quad$ PDF $(865 \mathrm{~K})$

Detection of magnesium ion concentration using fiber coupler based displacement sensor with concave mirror target Original Research Article Pages 37-43

M. Yasin, Samian, M. Khasanah

Abstract $\mid$ PDF (1443 K)

The influence of surface barriers on the photoemission characteristics of AIGaN photocathodes Original Research Article

Pages 44-52

Guanghui Hao, Junle Liu, Senlin Ke

Abstract | PDF $(1378 \mathrm{~K})$ 


\section{Optik}

Country Netherlands

Subject Area and

Category

Engineering

Electrical and Electronic Engineering

Materials Science

Electronic, Optical and Magnetic Materials

Physics and Astronomy

Atomic and Molecular Physics, and Optics

Publisher Elsevier BV

Publication type Journals

ISSN 00304026

Coverage 1968-1988, 1993-ongoing

Scope Optik publishes articles on all subjects related to light and electron optics and offers a survey on the state of research and technical development within the following fields: Optics: Optics design, geometrical and beam optics, wave optics - Optical and micro-optical components, diffractive optics, devices and systems - Photoelectric and optoelectronic devices - Optical properties of materials, nonlinear optics, wave propagation and transmission in homogeneous and inhomogeneous materials - Information optics, image formation and processing, holographic techniques, microscopes and spectrometer techniques, and image analysis - As well as other related topics. (source)

\section{Quartiles}

Atomic and Molecular Physics, and Optics

Electrical and Electronic Engineering

Electronic, Optical and Magnetic Materials

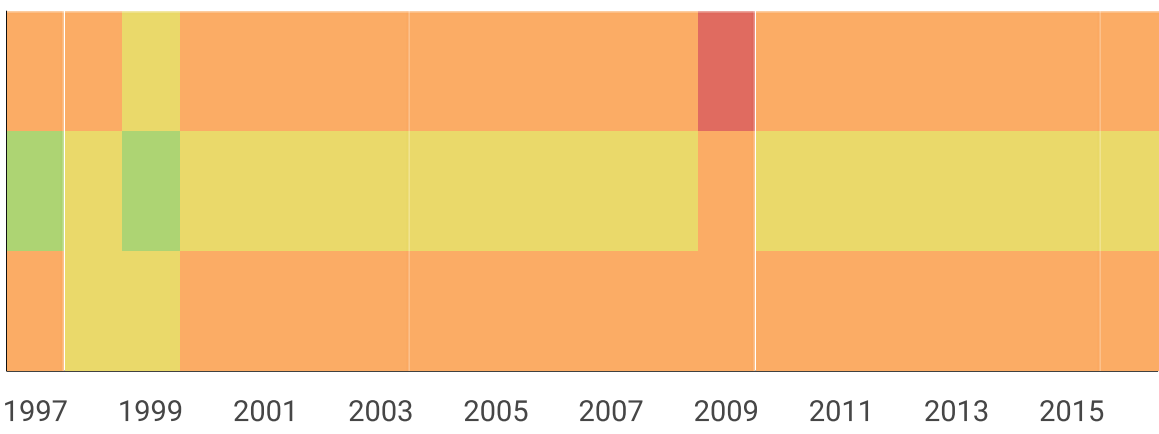

SJR 


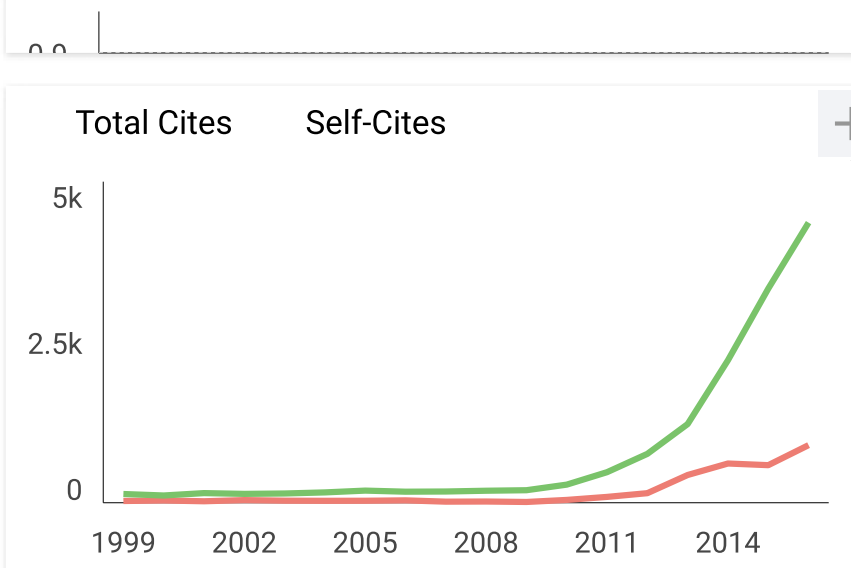

\section{External Cites per Doc}

Cites per Doc

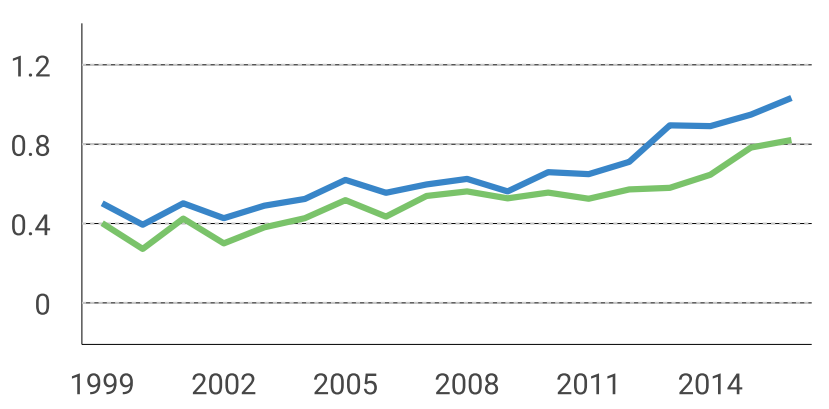

Citable documents Non-citable documents
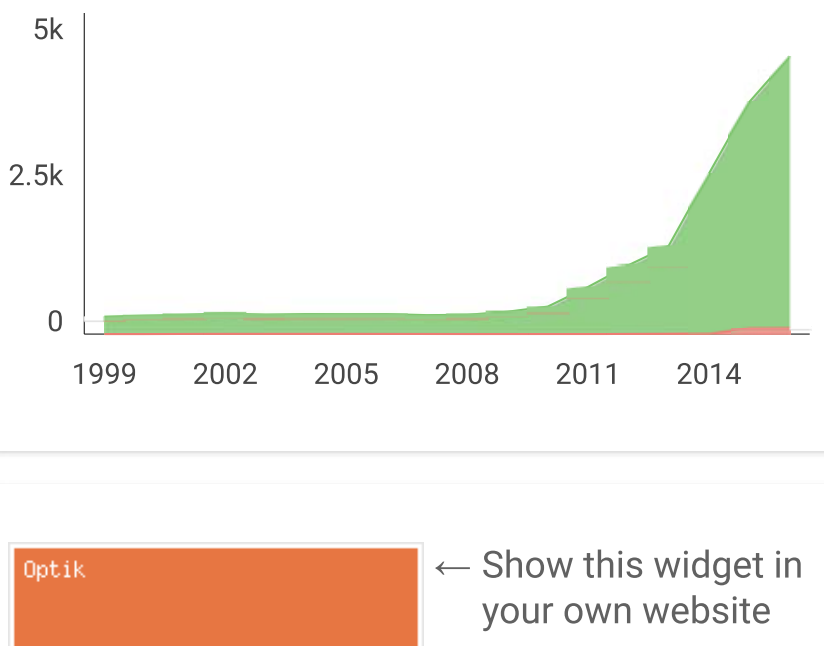

Indicator 2009-2016 Value

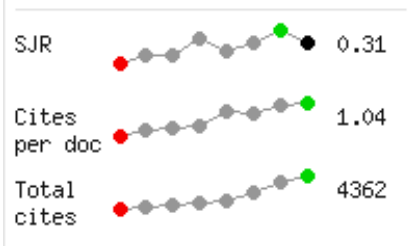

$\leftarrow$ Show this widget in your own website

Just copy the code below and paste within your html code:

<a href="http://www.scimagr

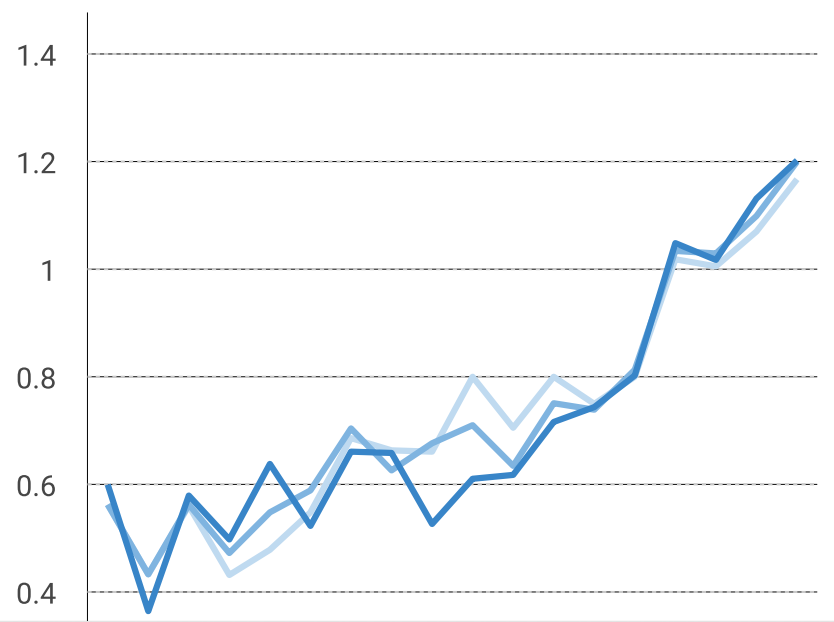

$\%$ International Collaboration

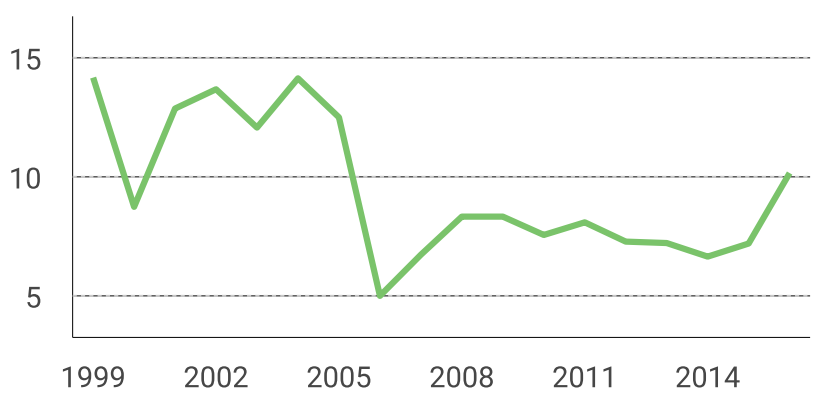

Cited documents Uncited documents

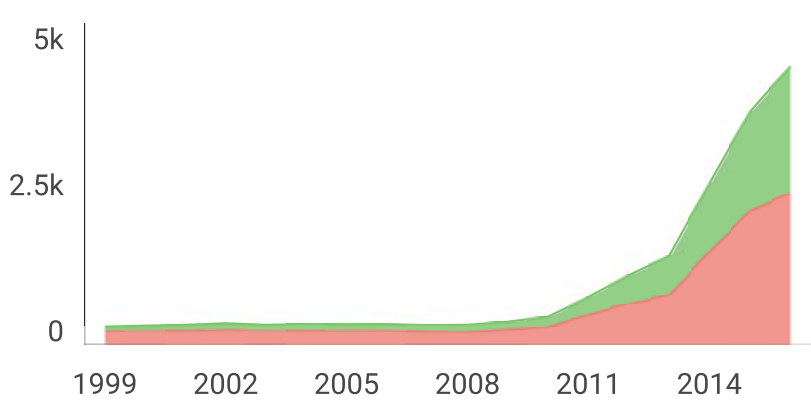




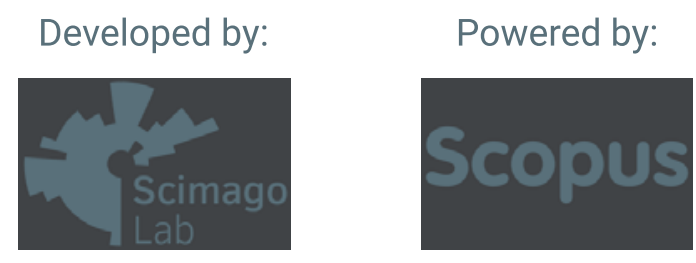

Follow us on Twitter

Scimago Lab, Copyright 2007-2017. Data Source: Scopus ${ }^{\circledR}$

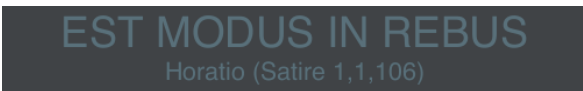




\title{
Detection of magnesium ion concentration using fiber coupler based displacement sensor with concave mirror target
}

\author{
M. Yasin ${ }^{\mathrm{a}, *}$, Samian $^{\mathrm{a}}$, M. Khasanah ${ }^{\mathrm{b}}$ \\ a Physics Department, Faculty of Science and Technology, Airlangga University, Surabaya 60115, Indonesia \\ b Chemistry Department, Faculty of Science and Technology, Airlangga University, Surabaya 60115, Indonesia
}

\section{A R T I C L E I N F O}

\section{Article history:}

Received 2 October 2017

Received in revised form 5 December 2017

Accepted 6 December 2017

\section{Keywords:}

Fiber coupler

Displacement sensor

Concave mirror

Refraction

\begin{abstract}
A B S T R A C T
Detection of magnesium ion concentration was successfully demonstrated using a fiber coupler based displacement sensor probe in conjunction with a concave mirror target. Analysis of peak voltage result, which was obtained by moving a sensing port displacement from a fixed concave mirror $(\mathrm{CM})$ was found to be useful to detect a magnesium ion concentration, which is filled in between the probe and target. The proposed sensor is capable for measuring magnesium ion with variation of concentration $0-5 \%$. The resolution of the sensor can be achieved at $0.2 \%$. It was also observed that the use of $\mathrm{CM}$ with a longer focal length improves the sensitivity of the sensor. The high sensitivity, the small size, the ease fabrication process, and the bio-compatibility of the proposed device are appealing characteristics that makes it ideal for practical bio-sensing applications.
\end{abstract}

(c) 2017 Elsevier GmbH. All rights reserved.

\section{Introduction}

Detections of refractive index and concentration of liquid solution have widely been reported using various fiber-optic sensors [1-5]. Owing to the intrinsic characteristics of the optical fiber, these sensors are in general simple to fabricate, compact, robust, immune to electromagnetic radiation, chemical and biological inert, and can be integrated in complex networks for real-time multiple parameter sensing. In recent years, many important advances were achieved in the fiber optic sensor technology; in terms of sensitivity, resolution, dynamic range, response time, signal to noise ratio and fabrication techniques. These advances have also promoted the development of optical fiber chemical sensors and biosensors [1,2]. For instance, the side-emitting technique was conducted using fiber optic micro-bend [3] and tapered fiber [4] to detect refractive index of chlorinated water and concentration of uric acid, respectively. Optical fibers coated with silver thin layer [5] and nanocomposite of ZnO-polypyrrol [6] were also used to detect concentration of uric acid and manganese ion based on surface plasmon resonance (SPR). Coreless fiber [7] and thin core fiber [8] connected to single mode fiber were used to detect concentration of sucrose and refractive index of solution, respectively.

A method based on displacement sensor can also be used to measure the concentration or refractive index of solution. For instance, a displacement sensor with a bundled fiber probe was capable of measuring refractive of solution [9], glucose concentration [10], calcium [11] and salinity [12]. This type of sensor works based on detection the output voltage as the displacement between sensor probe and mirror immersed in liquid of the solution sample is varied. Fiber coupler is an

\footnotetext{
* Corresponding author.

E-mail address: yasin@fst.unair.ac.id (M. Yasin).
} 


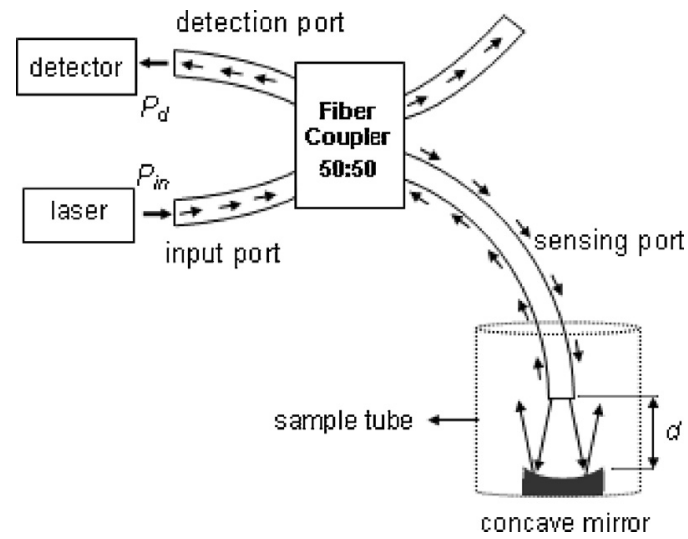

Fig. 1. Design of magnesium ion sensor using fiber coupler with concave mirror as reflector.

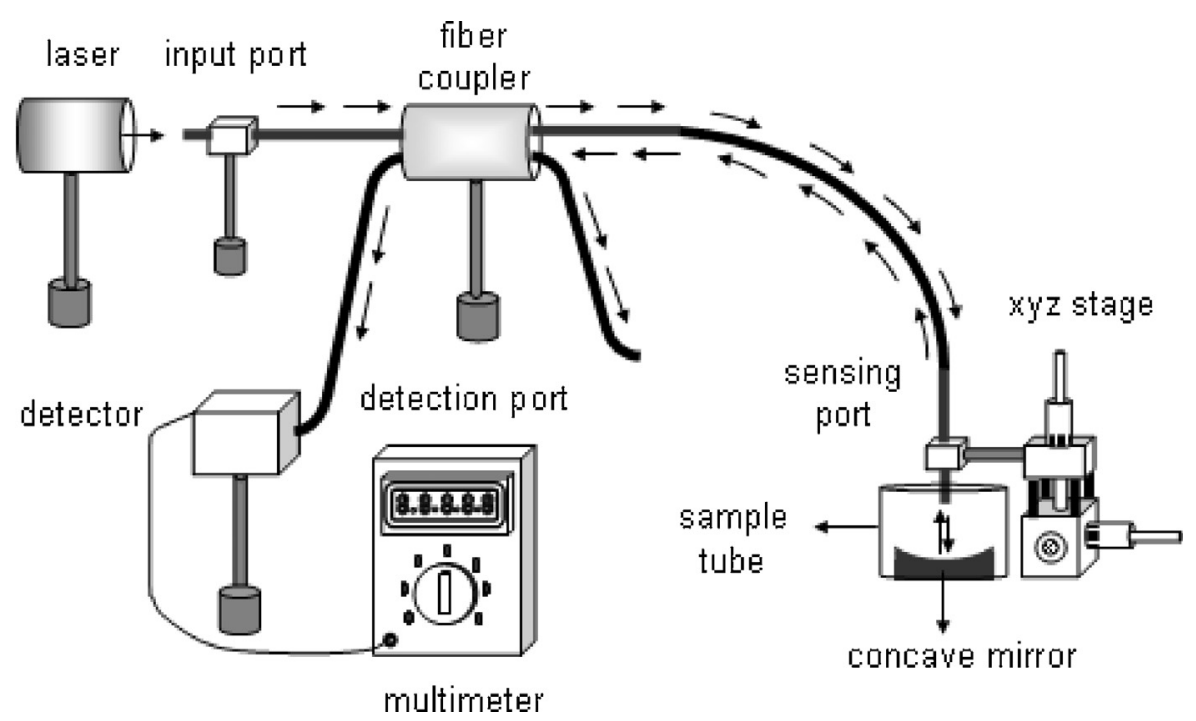

Fig. 2. Experimental setup for magnesium ion concentration sensor.

optical device that can be used to split a beam of light. It was used as a displacement sensor using a mirror as a target [13]. Based on the displacement sensor, fiber coupler was also employed as a level sensor [14] and a Rhodamine B concentration sensor [15] using a concave mirror (CM) as a target in conjunction with a green light source.

In this paper, we propose a new displacement sensor for measuring magnesium ion concentration using a fiber coupler as a probe and a concave mirror as a target. The magnesium ions detection is explored in this study since the ion is very important for the human in energy metabolism process, protein synthesis, muscle contraction, fat breakdown, DNA stability, and cell growth. Lack of magnesium ions in the body will affect health even can cause disease and cancer [16]. Therefore, the magnesium ions sensing device is becoming important especially for the food and beverage industry in order to meet the needs of magnesium ions that are perceived by the body.

\section{Working principle of the sensor}

Mechanism of magnesium ion detection using fiber coupler and concave mirror $(\mathrm{CM})$ is shown in Fig. 1. Light source of the laser is coupled to input port of the coupler, part of the output light beam from the sensing port $\left(P_{e}\right)$ enter to concave mirror. Medium between sensor port and CM is the testing sample. Partial of the reflected light beam from CM passed through a sensing port $\left(P_{b}\right)$ and then transmitted to the detection port $\left(P_{d}\right)$. The distance between sensing port and CM is attributed by $d$. If position of sensing port is changed in relation to CM, then the detected optical power as function of $d$ is given by [15];

$$
P_{d}=P_{0}\left(1-\exp \left(-\frac{2(d-f)^{2} / f^{2}}{\left(\frac{d^{2}-2 d f}{(d-f) a} \tan ^{-1} \sin ^{-1}\left(\frac{N A}{n}\right)+1\right)^{2}}\right)\right)
$$




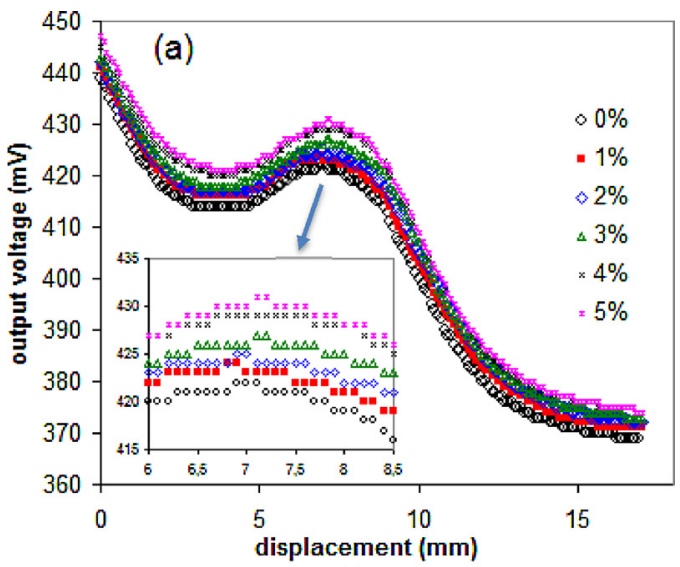

(a)

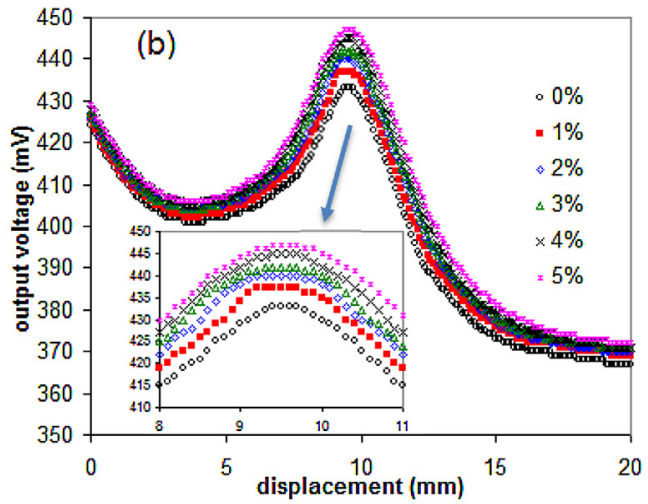

(b)

Fig. 3. Output voltage against the the displacement of sensing port as (a) CM 1 and (b) CM 2 was used as a target.

where $a, N A, f$ and $n$ is core of radius, numerical aperture, focal length of CM and refractive index of medium between sensing port and $\mathrm{CM}$, respectively. $\mathrm{P}_{0}$ can be written by equation as follows;

$$
P_{0}=1.15 c r(1-c r)\left(10^{-0.1 L e}-2 \times 10^{-0.1 D}\right)^{2} P_{\text {in }},
$$

where $c r, L_{e}$, and $D$ are coupling ratio, excess loss, and directivity of fiber coupler respectively. While $P_{\text {in }}$ optical power of the laser which is coupled to input port [13].

Optical power of laser is measured by an optical detector using digital voltmeter in term of output voltage $\left(P_{d}\right)$. Output power is proportional to output voltage. When Eq. (1) is simulated, we can obtain the curve showing the relationship between output voltage $(V)$ and $d$. The curve also indicates a peak voltage at a certain location. The value of $V_{p}$ increase with refractive index of medium. If we assumed that refractive index is a linear function to magnesium ion concentration in solution as a medium, then the test sample of magnesium ion concentration can be read from the peak voltage. Variation of position scanning between sensing port and concave mirror is used to obtained peak voltage.

\section{Experimental Setup}

The schematic experimental setup for the proposed magnesium ion concentration sensor is shown in Fig. 2. It consists of a He-Ne laser (with wavelength of $632.8 \mathrm{~nm}$ and maximum power of $10 \mathrm{~mW}$ ), fiber coupler and silicon detector. The fiber coupler probe was constructed from a multimode plastic fiber with structure $2 \times 2$, diameter of $1 \mathrm{~mm}, 50 / 50 \mathrm{splitting}$ ratio, $4 \mathrm{~dB}$ insertion loss and $1.6 \mathrm{~dB}$ excess loss. A CM with two different focal length 4.5 and $6 \mathrm{~mm}$ was used as reflector. CM was placed on the bottom sample tube with diameter of $4 \mathrm{~cm}$ and $3 \mathrm{~cm}$ height.

The positioning of the fiber coupler sensing port was accomplished by mounting it on a micro-displacement meter, which was rigidly attached to a vibration free table. Light from the fiber coupler input port was coupled into the sensing port. The signal from sensing port was measured by moving the probe away from the zero point, where the reflective surface of concave mirror and the probe were in close contact. The signal from the optical detector was converted into voltage and was measured by a digital voltmeter. The output intensity was measured by changing the position of the fiber coupler sensing port, which functions as probe from 0 to $15 \mathrm{~mm}$ in step of $100 \mu \mathrm{m}$. The measurements were carried out for magnesium 

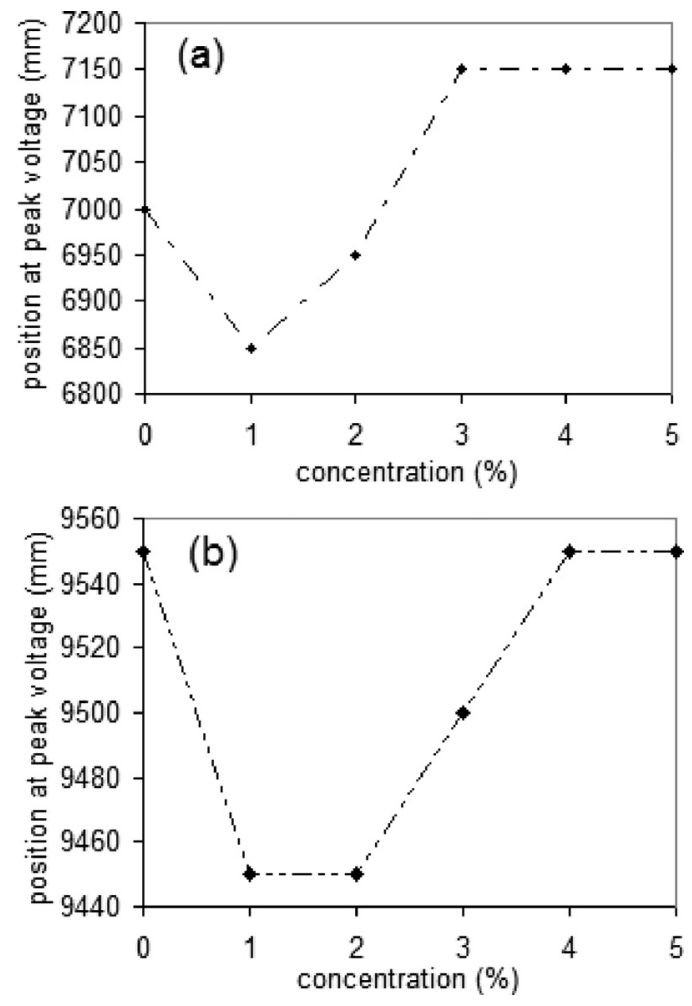

Fig. 4. Position at peak voltage versus magnesium ion concentration for (a) CM 1 and (b) CM 2 .

ion with concentrations of 1,2,3,4 and 5\%. During the experiment, temperature was kept constant to that error due to temperature fluctuation is negligible.

\section{Result and Discussion}

Detection of magnesium ion concentration was obtained by monitoring the output voltage while the displacement between the sensing port and CM is varied. The initial position of the sensing port is about $2 \mathrm{~mm}$ above the center of the $\mathrm{CM}$. The curves related to the output voltage as function of displacement sensing port was measured for two different CMs for comparison purpose. The results are analyzed and plotted as shown in Fig. 3. Figs. 3(a) and (b) shows the displacement curves with CM1 and CM2, which has a focal length 6 and $12 \mathrm{~mm}$, respectively. The measurement error for both curves is obtained at around $0.6 \mathrm{mV}$. It is shown that the peak voltage position was obtained at around $7.0 \mathrm{~mm}$ and $9.5 \mathrm{~mm}$ for the displacement curves with CM1 and CM2, respectively. It is worthy to note that CM 1 and CM 2 has a diameter of $9.0 \mathrm{~mm}$ and $12.0 \mathrm{~mm}$, respectively. In the experiment, the starting position of sensing port was at $2.0 \mathrm{~m}$ from surface of concave mirror. Compare to reference of 12 , the main novelty of the proposed setup is applying fiber coupler probe as element sensing. In this probe, we have a first peak voltage at very close distance, therefore the sensor is suitable for a shorter distance measurement.

Figs. 4(a) and (b) show the peak voltage position at various magnesium ion concentrations for CM1 and CM2 target respectively. It is shown from both figures that peak voltage position changes irregularly with the variation of magnesium ion concentration. Therefore, it is difficult to predict magnesium ion concentration using the peak voltage position.

Correlation between peak voltage value and magnesium ion concentration is shown in Fig. 5. It indicates a linear trend relation between the peak voltage and magnesium ion concentration. The linearity is more than $99 \%$. Therefore, we can determine value of concentration based on value of peak voltage. Slope of the curve in Fig. 5 defines sensitivity of the sensor, which indicates CM2 produces a better sensitivity than that of CM1. This is attributed to the longer focal length that allows the sensing fiber port to collect more light and increases the sensitivity. The refractive index of various magnesium ion concentrations was also measured using Abbe refractometer and the result is summarized in Fig. 6 . The result shows that the refractive index is linearly correlated with the concentration. Therefore, this approach can be used for detection of magnesium ion concentration.

The stability of the sensor was then tested for $720 \mathrm{~s}$ for each interval $30 \mathrm{~s}$ and the result is presented in Fig. 7. Fig. 7 shows the sensor has a high stability with standard of deviation is $0.4 \mathrm{mV}(0.1 \%)$ and $0.5 \mathrm{mV}(0.11 \%)$ for $\mathrm{CM}$ 1 and CM 2, respectively. Resolution of the sensor is defined by ratio of standard deviation over sensitivity (slope of the linear curve). Overall, performance of the proposed magnesium ion concentration sensor using fiber cou- 

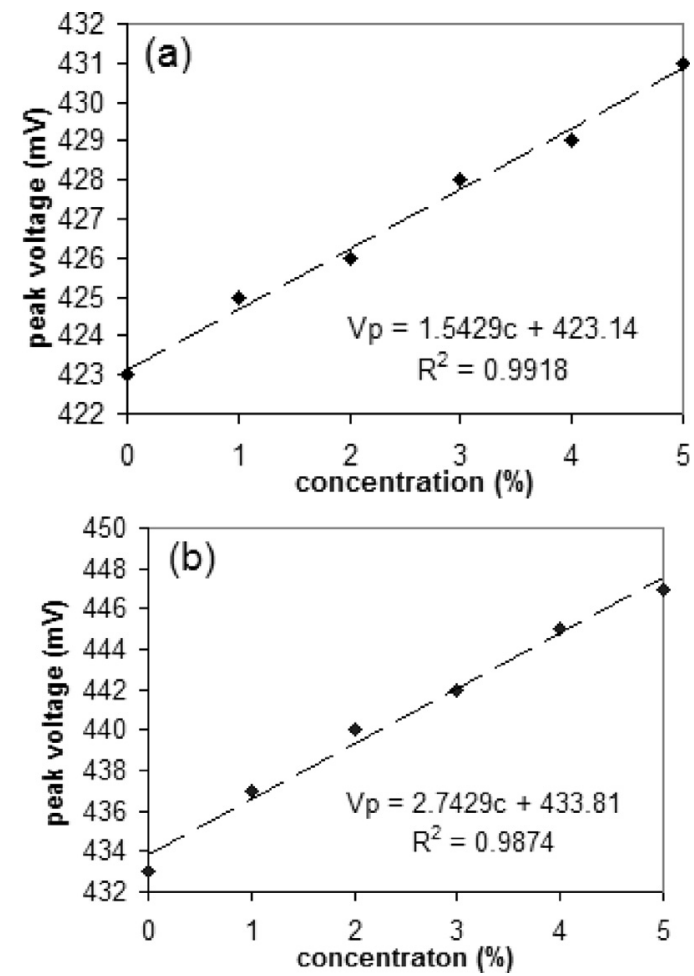

Fig. 5. Peak voltage as a function of magnesium ion concentration for (a) CM 1 and (b) CM 2 .

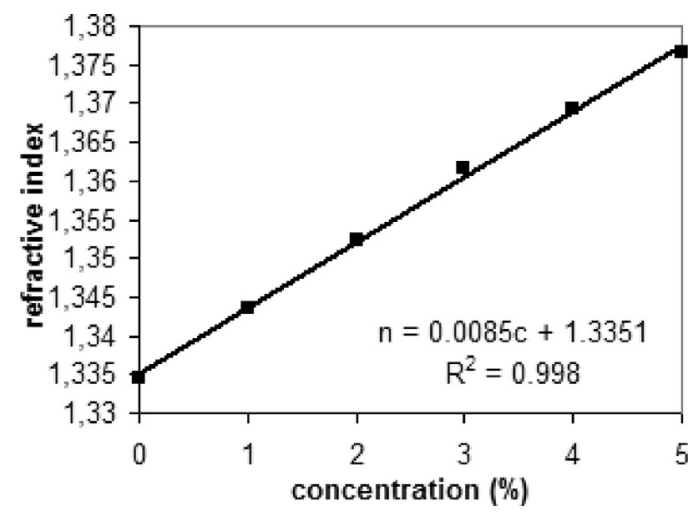

Fig. 6. Result of refractive index measurement versus a magnesium ion concentration.

Table 1

Performance of the sensor for magnesium ion detection with CM 1 and CM 2.

\begin{tabular}{lll}
\hline Parameters & Value & \\
\cline { 2 - 3 } & CM 1 & CM 2 \\
\hline Sensor range (\%) & $0-5$ & $0-5$ \\
Linear region (\%) & $0-5$ & $0-5$ \\
Sensitivity (mV/\%) & 1.54 & 2.75 \\
Resolution (\%) & 0.3 & 0.2 \\
\hline
\end{tabular}

pler probe for CM 1 and CM 2 is summarized in Table 1. Table 1 shows that sensitivity of the sensor with CM 1 is higher than sensor with CM 2, therefore the sensor resolution with CM 2 is lower than CM 1 (1/1.5 times). The results indicate that fiber coupler based displacement sensor can be used for detection of magnesium ion concentration. During the experiment, the error due to this temperature variation is negligible as the temperature is kept constant at $25^{\circ} \mathrm{C}$. 

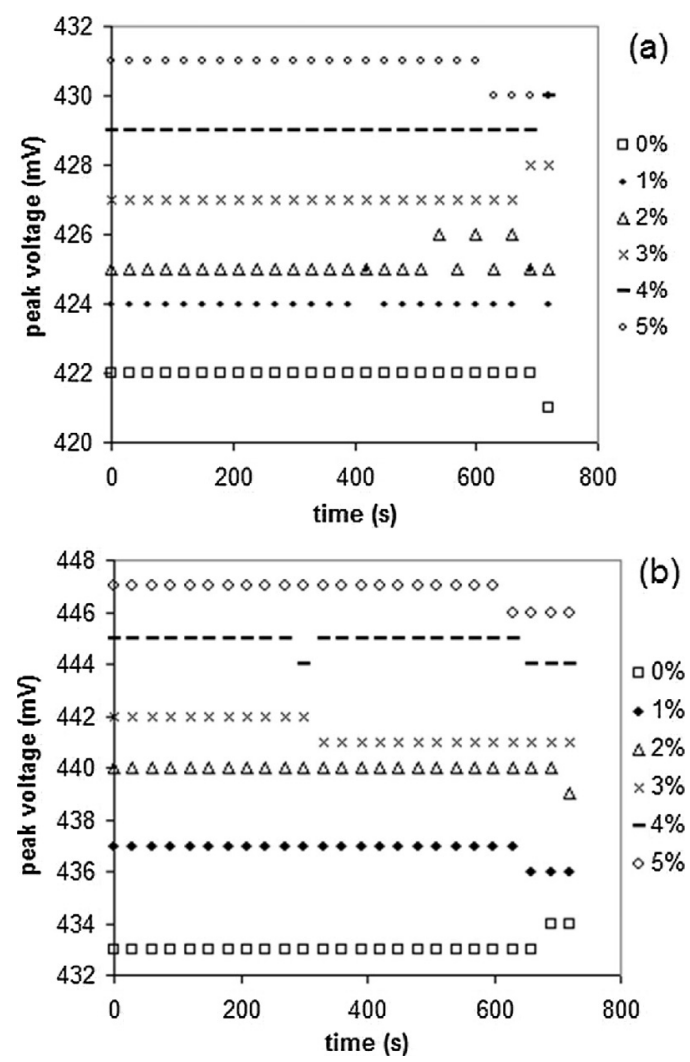

Fig. 7. Stability of the sensor for (a) CM 1 and (b) CM 2.

Based on a simple calculation, a response time or bandwidth of optical detector is used in this experiment can be found at $0.175 \mathrm{kHz}$.

\section{Conclusion}

Fiber optic coupler based displacement sensor configured with CM was proposed and demonstrated for detection of magnesium ion concentration. The results shows that a peak voltage of the sensor is linear to the concentration with the best resolution is obtained at $0.2 \%$ and range of $0-5 \%$. The use of $\mathrm{CM}$ with a longer focal length improves the sensitivity of the sensor. The simplicity and low cost of the sensor make it suitable for various industrial applications.

\section{Acknowledgement}

This work was supported by the Airlangga University through “Mandat Grant” No: 569/UN3/2017.

\section{References}

[1] Xu-dong Wang, Otto S. Wolfbeis, Fiber-optic chemical sensors and biosensors (2013-2015), Anal. Chem. 88 (2016) $203-227$.

[2] Christophe Caucheteur, Tuan Guo, Jacques Albert, Review of Plasmonic Fiber optic biochemical sensors: improving the limit of detection, Anal. Bioanal. Chem. 407 (2015) 3883-3897.

[3] NibuA. George, AnuMaria Paul, M.S. Saranya, Microbend Fiber optic detection of continuously varying refractive index of chlorinated water, Optik 125 (2014) 301-303.

[4] H.R.A. Rahim, N. Irawati, H.A. Rafaie, H. Ahmad, S.W. Harun, R.M. Nor, detection Of different concentrations Of uric acid using tapered silica optical sensor coated with zinc oxide (Zno), Jurnal Teknologi 74 (8) (2015) 55-58.

[5] Ravi Kant, Rana Tabassum, BanshiD. Gupta, Fiber optic SPR based uric acid biosensor using uricase entrapped polyacrylamide gel, IEEE 28 (19) (2016) 2050-2053.

[6] Rana Tabassum, BanshiD. Gupta, Fiber optic manganese ion using SPR nanocomposite of ZnO-polypyrrol, Sens. Actuators B 220 (2015) $903-909$.

[7] WuroodAbdulkhaleq Khaleel, AbdulHadiM. Al-Janabi, High-sensitivity sucrose erbium-doped fiber ring laser sensor, Opt. Eng. 56 (2) (2017) 026116.

[8] Jiangtao Zhou, Yiping Wang, Changrui Liao, Sun Bing, Jun He, Guolu Yin, Shen Liu, Zhengyong Li, Guanjun Wang, Xiaoyong Zhong, Jing Zhao, Intensity modulated refractive index sensor based on optical fiberMichelson Interferometer, Sens. Actuators B 208 (2015) 315-319.

[9] Gobi Govindan, Dillibabu Srinivasan Gokul Raj, Sastikumar, Measurement of refractive index of liquids using fiber optic displacement Sensors, J. Am. Sci. 5 (2) (2009) 13-17.

[10] S. Binu, V.P. Mahadevan Pillai, V. Pradeepkumar, B.B. Padhy, C.S. Joseph, N. Chandrasekaran, Fibre optic glucose sensor, Mater. Sci. Eng. C 29 (2009) 183-186. 
[11] M. Yasin, S. Soelistiono, Y.G. Yhun Yhuwana, M. Khasanah, H. Arof, N. Irawati, S.W. Harun, Intensity based optical fiber sensors for calcium detection, J. Optoelectron. Adv. Mater. 9 (9-10) (2015) 1185-1189.

[12] HusnaAbdul Rahman, SulaimanWadi Harun, M. Yasin, Harith Ahmad, fiber-optic salinity sensor using fiber-optic displacement measurement with flat and concave mirror, IEEE J. Sel. Top. Quant. Electron. 18 (5) (2012) 1529-1533.

[13] Samian, Y.H. Pramono, A.Y. Rohedi, F. Rusydi, A.H. Zaidan, Theoretical and experimental study of fiber-optic displacement sensor using multimode fiber coupler, J. Optoelectron. Adv. Mater. Rapid Commun. 3 (5) (2009) 411-414.

[14] Samian, G.Y.Y. Yhosep, A.H. Zaidan, Herlik Wibowo, Gasoline level sensor based on displacement sensor using fiber coupler, Measurement 58 (2014) $342-348$.

[15] Samian, A.H. Zaidan, Moh. Yasin, Detection of Rhodamine B levels in distilled water based on displacement sensor using fiber coupler and concave mirror, J. Optoelectron. Adv. Mater. 18 (11-12) (2016) 988-992.

[16] K.P. Schlingmann, M. Konrad, H.W. Seyberth, Genetics of hereditary disorders of magnesium Homeostatus, Pediatr. Nephrol. 19 (1) (2004) 13-15. 